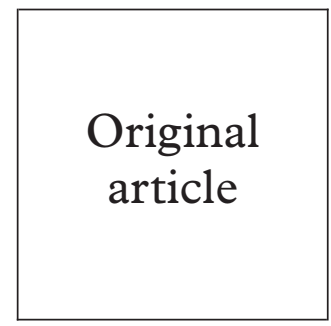

\title{
Trichomonad invasion of the mucous layer requires adhesins, mucinases, and motility
}

\author{
Michael W Lehker, Daniel Sweeney
}

Background/objective: Trichomonas vaginalis, the causal agent of trichomonosis, is a flagellated parasitic protozoan that colonises the epithelial cells of the human urogenital tract. The ability of $T$ vaginalis to colonise this site is in part a function of its ability to circumvent a series of non-specific host defences including the mucous layer covering epithelial cells at the site of infection. Mucin, the framework molecule of mucus, forms a lattice structure that serves as a formidable physical barrier to microbial invasion. The mechanism by which trichomonads traverse the mucous covering is unknown. Proteolytic degradation of mucin, however, may provide for a mechanism to penetrate this layer. The goal, therefore, was to determine how trichomonads cross through a mucous layer.

Methods: Secreted trichomonad proteinases were analysed for mucinase activity by mucin substrate-sodium dodecyl sulphate-polyacrylamide gel electrophoresis. The importance of trichomonad mucinases for traversing the mucous layer was examined on an artificial mucin layer in invasion chambers. Adherence to mucin and tissue culture cells was measured using a microtitre plate assay.

Results: Trichomonad isolate 24402 secreted five proteinases when incubated in PBS. All five proteinases were shown to possess mucinase activity. These mucinases were able to degrade bovine submaxillary mucin and to a lesser extent porcine stomach mucin. These enzymes were active over a $\mathrm{pH}$ range of 4.5-7.0 and were inhibited with cysteine proteinase inhibitors. Furthermore, $T$ vaginalis was shown to bind to mucin possibly via a lectin-like adhesin. Adherence to mucin was increased threefold when parasites were grown in iron deficient medium. Adherence to soluble mucin prevented attachment to HeLa cells. Proteinase activity, adherence, and motility were required for trichomonads to traverse a mucin layer in vitro.

Conclusions: These results show that trichomonads can traverse the mucous barrier first by binding mucin followed by its proteolytic degradation. The data further underscore the importance of trichomonad proteinases in the pathogenesis of trichomonosis. Finally, this study suggests that interference with trichomonad mucin receptors and proteinases may be a strategy to prevent colonisation by this parasite.

(Sex Transm Inf 1999;75:231-238)

Keywords: trichomonads; mucous layer; adhesins; mucinases; motility

\section{Introduction}

Trichomonas vaginalis is the microbial agent responsible for trichomonal vaginitis, a major sexually transmitted disease worldwide. ${ }^{1}$ Transmission of this parasite occurs almost exclusively by sexual contact ${ }^{1}$ and thus the mucous layer of the genital tract is the first host surface encountered by trichomonads. The major proteinaceous constituent of mucus is mucin. ${ }^{2}$ Mucins are large glycoproteins varying in size from approximately 1 to $35 \times 10^{6} \mathrm{Da}$. Portions of the peptide backbone of mucin are heavily glycosylated and usually resistant to proteolytic cleavage. Intermolecular disulphide bridges in conjunction with hydrophobic interaction give mucin its gel-like property. ${ }^{3}$ The size of mucin, its gel-like property, and its heavy glycosylation make it a formidable physical barrier to pathogens attempting to colonise the underlying epithelium. Thus, the outcome of the interaction of trichomonads with the host mucous layer will determine whether $T$ vaginalis will be successful in colonising a new host.

$T$ vaginalis possesses several factors, in particular adhesins and proteinases, that may be involved in breaching the mucous layer. Adherence to host surfaces has been shown to be an early and critical step in microbial pathogenesis. ${ }^{4}$ Cytoadherence in trichomonads to the vaginal epithelial mucosa is mediated by at least four surface adhesin proteins. ${ }^{5}$ Furthermore, trichomonad proteinase activity appears to be necessary for cytoadherence to occur. ${ }^{6}$ Adhesin proteins are upregulated during times of abundant iron sources, such as during menstruation. It has been suggested that increased adherence during menses prevents loss of the parasite. ${ }^{7}$ Likewise, it can be envisaged that an adherence mechanism for mucin may allow trichomonads to gain a temporary foothold before penetration of the mucous layer and ultimate parasitism of the underlying epithelial cells.

Trichomonads possess numerous cysteine proteinases, most of which are secreted during axenic growth. ${ }^{8}$ This has also been shown to occur in vivo since trichomonad proteinases have been detected in vaginal secretions of infected individuals. ${ }^{9}$ In addition, it is likely that the proteinases are active in vivo as the vaginal milieu is favourable for activation of the 
trichomonad proteinases. ${ }^{10}$ Importantly, it has also been suggested that these proteinases can degrade a variety of host substrates such as haemoglobin, laminin, and immunoglobulins. ${ }^{11}$ Indeed, studies using synthetic substrates confirm the broad substrate specificity of these proteinases. ${ }^{12}$ Since trichomonad proteinases are able to degrade a variety of substrates, we hypothesised that they may also degrade mucin and thus allow this parasite to traverse the mucous layer.

In this study we examined the interaction of $T$ vaginalis with mucin and showed that adherence, mucinolytic activity, and motility are all required for trichomonads to successfully penetrate a mucous layer.

\section{Materials and methods}

CULTURES, LYSATE PREPARATION, AND PROTEINASE SECRETION

$T$ vaginalis isolates used in this study were kindly provided by J F Alderete (University of Texas Health Science Center at San Antonio, San Antonio, TX, USA). All trichomonads were cultured in trypticase-yeast extractmaltose (TYM) medium supplemented with $5 \%$ heat inactivated horse serum. ${ }^{13}$ High iron TYM medium was made by adding ferrous ammonium sulphate hexahydrate (Sigma Chemical Company, St Louis, MO, USA) to a final concentration of $0.1 \mathrm{mM} .{ }^{14}$ This concentration of iron has been shown previously to induce an iron replete phenotype. ${ }^{15}$ Low iron TYM medium was prepared by the addition of the ferrous iron chelator ferrozine (Sigma) to a final concentration of $0.125 \mathrm{mM}$. Sodium dodecyl sulphate-polyacrylamide gel electrophoretic (SDS-PAGE) analysis of trichomonad protein patterns under these growth conditions, demonstrated the induction of an iron deficient phenotype. ${ }^{15}$

Cell lysates were prepared using organisms from mid to late logarithmic growth phase. Parasites were washed three times in phosphate buffered saline (PBS) $(16 \mathrm{mM} \mathrm{KCl}, 61 \mathrm{mM}$ $\mathrm{Na}_{2} \mathrm{HPO}_{4}, 8 \mathrm{mM} \mathrm{KH} \mathrm{PO}_{4}, 0.82 \mathrm{M} \mathrm{NaCl}, \mathrm{pH}$ $7.2)$ and resuspended in SDS-PAGE sample buffer $^{16}$ (125 mM TRIS-HCl, pH 6.8, 4\% SDS, $20 \%$ glycerol, and $0.1 \%$ bromophenol blue) in the absence of $\beta$ mercaptoethanol at a concentration of $5 \times 10^{7}$ cells $/ \mathrm{ml}$. Parasite secretions were prepared by resuspending washed cells $\left(2 \times 10^{7} / \mathrm{ml}\right)$ in PBS supplemented with maltose $(5 \mathrm{~g} / \mathrm{l})$. Trichomonads were then incubated at $37^{\circ} \mathrm{C}$ for 2 hours. At the conclusion of the incubation period, parasites were removed by centrifugation and the supernatant was filtered through a $0.2 \mu \mathrm{M}$ syringe filter to remove any cell debris. This protocol resulted in less than $1 \%$ lysis of parasites as measured by lactate dehydrogenase (LDH) release. ${ }^{17}$ Supernatants were then concentrated to $1 / 10$ th volume by lyophilisation and dialysed against several changes of double deionised water. Supernatants were used either immediately or stored at $-70^{\circ} \mathrm{C}$. Storage of supernatants for up to 4 weeks did not affect the proteinase pattern, as also has been noted by others. ${ }^{11}$
SDS-PAGE AND SUBSTRATE GEL ELECTROPHORESIS Degradation of mucin was followed by SDSPAGE analysis. Briefly, $100 \mu \mathrm{l}$ of mucin (1 $\mathrm{mg} / \mathrm{ml}$ ) were co-incubated with concentrated supernatant $(100 \mu \mathrm{l})$ and incubated at $37^{\circ} \mathrm{C}$ for various periods of time (0.25-24 hours). Aliquots of $20 \mu \mathrm{l}$ were then removed and loaded onto individual wells of a SDS-PAGE consisting of a $4 \%$ and $8 \%$ stacking and separating gel, respectively. Gels were then stained with Schiff's reagent (Sigma) as recommended by the manufacturer. This stain specifically detected mucins and staining of supernatant components was not observed in control gels (data not shown).

Detection of mucinases was carried out by substrate SDS-PAGE. ${ }^{81819}$ Supernatants or lysates were applied to individual lanes of SDS-PAGE ( $8 \%$ ) copolymerised with gelatin, bovine submaxillary mucin (BSM), or porcine stomach mucin (PSM) at $0.5 \mathrm{mg} / \mathrm{ml}$ of separating gel. The stacking gel $(4 \%)$ contained no substrates. Proteins were then electrophoresed at $50 \mathrm{~V}$ in a minigel apparatus (BioRaD Laboratories, Hercules, CA, USA). After electrophoresis, gels were incubated in developing buffer ( $1 \mathrm{mM}$ DTT, $140 \mathrm{mM}$ sodium acetate, $2.5 \%$ Triton $\mathrm{X}-100, \mathrm{pH} 6.0$ ) overnight at $37^{\circ} \mathrm{C}$. Gels were then washed twice with distilled water and stained with Coomassie brilliant blue overnight. Next day, gels were destained for 4 hours in a solution of $7 \%$ acetic acid and 5\% methanol.

\section{MUCIN ADHERENCE ASSAY}

Binding of trichomonads to mucin was analysed by a modified microtitre plate assay. ${ }^{6}$ Mucin dissolved in PBS $(10 \mu \mathrm{g} / \mathrm{ml})$ was absorbed onto polystyrene microtitre plates. Coated wells were then washed with trichomonad binding medium (0.5× CRML tissue culture medium). Washed parasites, 100 $\mu \mathrm{l}$, suspended in binding medium $\left(2 \times 10^{7}\right.$ cells/ $\mathrm{ml}$ ) were added to each well and incubated at $37^{\circ} \mathrm{C}$. After 45 minutes, unbound cells were removed by aspiration and wells were washed three times with $200 \mu \mathrm{l}$ of warm binding medium. Next, $50 \mu \mathrm{l}$ of $1 \%$ Triton X-100 was added to each well. LDH activity of each well was then measured using CytoTox 96 Nonradioactive Cytotoxicity Assay Kit from Promega. LDH activity correlated with cell number and was linear over a range of $1 \times 10^{4}$ to $1 \times 10^{6}$ cells.

HELA CELL ADHERENCE ASSAY

HeLa cells, obtained from ATCC, were routinely maintained in Dulbecco modified minimal essential medium supplemented with $10 \%$ fetal bovine serum, $100 \mathrm{U}$ of penicillin $/ \mathrm{ml}$, and $100 \mu \mathrm{g}$ of streptomycin $/ \mathrm{ml}$. Cells were incubated under $7 \%$ carbon dioxide in air at $37^{\circ} \mathrm{C}$. Medium was changed daily and cells were passaged when monolayers reached confluency into either $25 \mathrm{~cm}^{2}$ flask for maintenance or into individual wells in 96 well plates for adherence studies. For adherence studies $100 \mu \mathrm{l}$ of a parasite $\left(2 \times 10^{7}\right.$ cells $\left./ \mathrm{ml}\right)$ suspension in binding medium, with or without mucin, was added to a confluent HeLa cell monolayer 
in microtitre wells. Tissue culture cells and trichomonads were then co-incubated for 1 hour at $37^{\circ} \mathrm{C}$. Non-adherent parasites were then removed by gentle washing of the monolayer. Adherence to HeLa cells was then quantitated as described above for mucin adherence.

\section{INVASION ASSAY}

Microporous $(8 \mu \mathrm{M})$ invasion chambers (Collaborative Biomedical Products, MA, USA) were coated with $300 \mu \mathrm{l}$ of $0.5 \%$ low melting agarose containing $12.5 \%$ mucin $(\mathrm{w} / \mathrm{w})$. Once gelling had occurred, $2 \times 10^{7}$ parasites in $1 \mathrm{ml}$ of TYM medium were added to the invasion chamber. The invasion chambers were placed into microtitre wells each containing $2 \mathrm{ml}$ of TYM $10 \%$ serum. The plate was then incubated at $37^{\circ} \mathrm{C}$ for various periods of time. To determine if trichomonads were able to cross the agarose-mucin gel, individual chambers were removed periodically and the lower wells examined by light microscopy. Numbers of trichomonads in the lower chamber were calculated by counting the number of cells in 20 randomly chosen microscopic fields.

\section{Results}

T VAGINALIS ADHERES TO MUCIN

Adherence to mucin, the major component of the mucous layer, ${ }^{2}$ may be essential in establishing a foothold in the host. To test if $T$ vaginalis binds mucin, parasites were grown in high and low iron medium ${ }^{15}$ and added to microtitre wells coated with BSM and PSM. The results in figure 1 show that trichomonads specifically adhere to mucin. Adherence to BSM and PSM was identical when parasites were grown under high iron conditions. When organisms were grown under low iron conditions, however, adherence to PSM but not BSM increased threefold (fig 1A). This result may suggest an iron dependent induction of a PSM specific adhesin. Microscopic observation of trichomonad adherence to coated and uncoated wells further substantiated the specific binding to mucin. Figure 1B shows that adherence to plastic surfaces (B2) was negligible when compared with adherence to mucin coated plastic surfaces (B1). Levels of adherence to bovine serum albumin coated wells was similar to that of uncoated wells (data not shown).

Since mucin binding may protect against adherence of micro-organisms to the underlying epithelium ${ }^{20}$ we next determined whether trichomonad binding of mucin abrogates cytoadherence. Trichomonads were allowed to interact with HeLa cells in the presence or absence of various amounts of mucin. As shown in figure 2, mucin inhibited binding to HeLa cells in a dose dependent fashion. Maximal inhibition of $68 \%$ was observed when 1 $\mathrm{mg} / \mathrm{ml}$ mucin was present during the coincubation of trichomonads with HeLa cells. These results suggest that mucin serves as an important host defence against trichomonad colonisation.
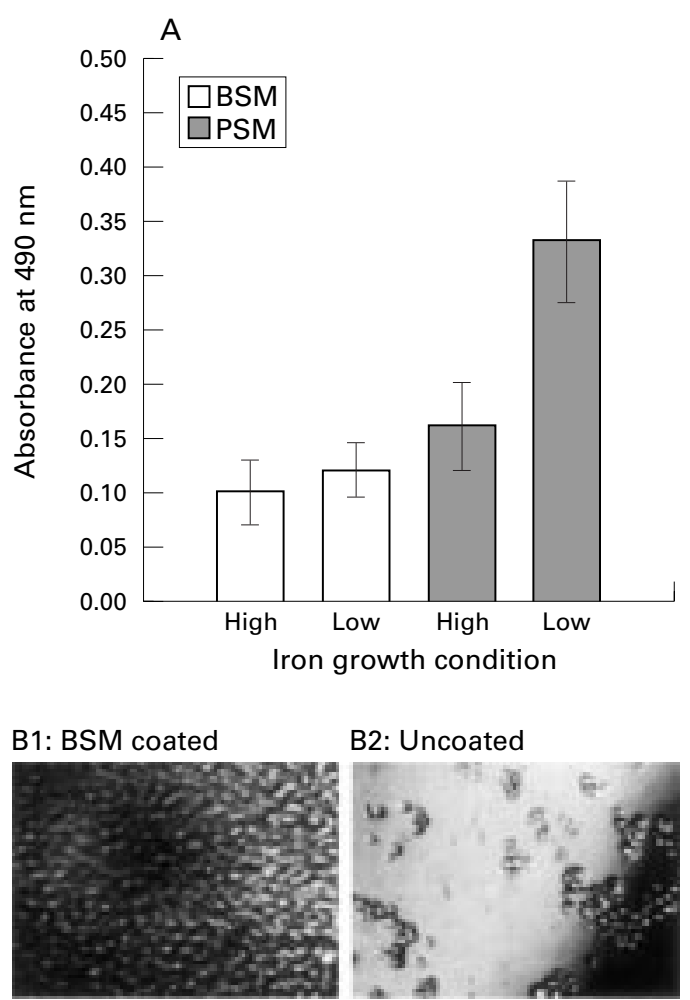

Figure 1 Adherence of $T$ vaginalis to mucin coated microtitre well. (A) The level of binding and standard deviation of high and low iron grown trichomonads to wells coated with bovine submaxillary mucin (BSM) and porcine stomach mucin (PSM). All experiments were done in triplicate and performed on at least six different days. (B) A comparison of trichomonad adherence to a BSM coated well and to plastic alone.

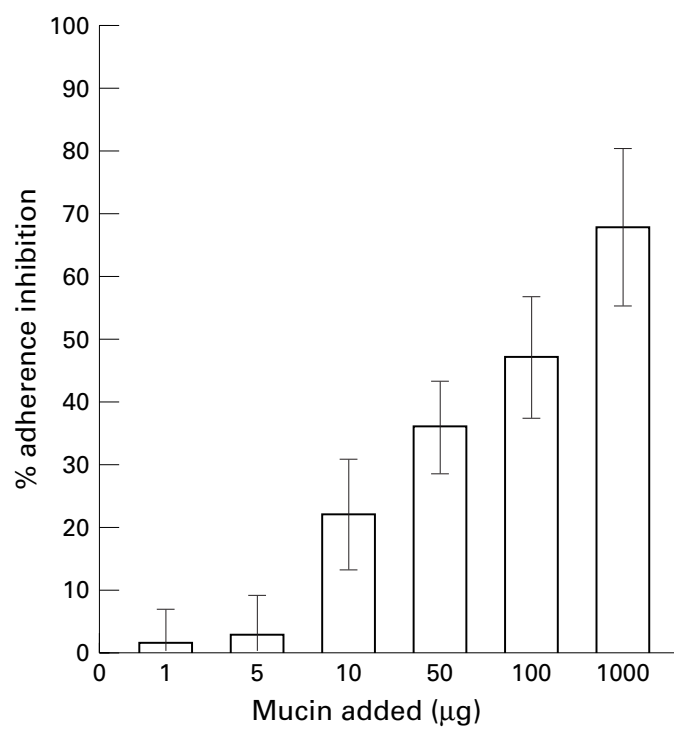

Figure 2 Inhibition of trichomonad cytoadherence to HeL a cells by mucin. Trichomonads were co-incubated with HeLa in 96 well microtitre plates in the presence of various amounts of mucin. Adherence to HeLa cell was quantitated by LDH assay. Adherence to HeLa cells in the absence of mucin was set as $100 \%$ adherence.

\section{TRICHOMONAD CYSTEINE-LIKE PROTEINASES} DEGRADE MUCIN

In vivo, trichomonads adhere tightly to vaginal epithelial cells, demonstrating their ability to breach the mucous layer. Degradation of mucin by proteinases appear to be major mechanism by which micro-organisms can gain access to the underlying epithelium. Numerous trichomonad proteinases, most of 

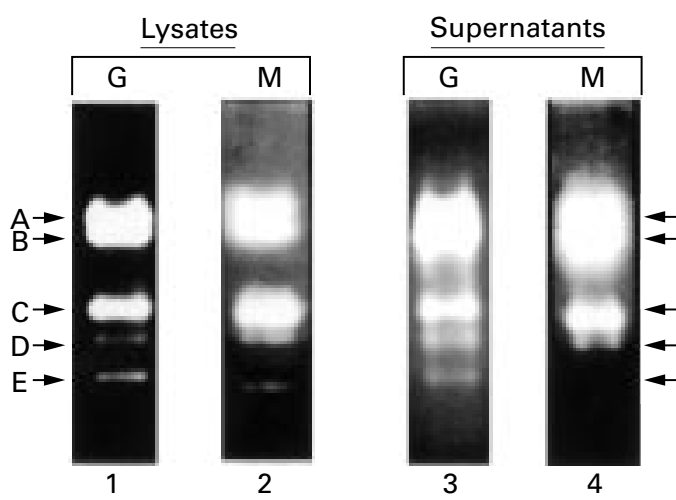

Figure 3 Degradation of bovine submaxillary mucin by trichomonad cysteine proteinases. Trichomonad cell associated (lysate) or extracellular (supernatant) proteinases were examined by substrate gel electrophoresis with gelatin $(G)$ or mucin $(M)$ copolymerised with acrylamide. Cleared areas represent digestion of the substrate.

which are secreted during axenic growth, have been described. ${ }^{8}{ }^{12}$ In order to determine whether these proteinases play a role in the penetration of the mucous layer, we ascertained if any trichomonad proteinases could degrade mucin. Trichomonad lysates and supernatants were analysed by substrate gel electrophoresis (fig 3). Subsequent negative staining revealed five clearings on the gel which were indicative of mucinase activity (fig 3, lane 2). Five mucinases of identical molecular weight were found to be present in cell lysates as well as in supernatants (fig 3, lanes 2 and 4). Furthermore, the proteinase patterns on substrate gels co-polymerised with either mucin or gelatin were also identical (fig 3, lanes 1, 2; lanes 3,4$)$. These results suggest that secreted mucinases are not a subset of the organism's proteinase repertoire.

To characterise these secreted mucinases, trichomonad supernatant samples were electrophoresed on BSM-substrate gels and activated in the absence or presence of protease inhibitors. Table 1 summarises the inhibition profiles of secreted trichomonad mucinases. Serine protease and metalloprotease inhibitors had no effect on mucinase activity. In contrast, cysteine protease inhibitors and some serine protease inhibitors, which are also known to inhibit some cysteine proteases, completely abolished mucinase activity. Interestingly, the cysteine protease inhibitor E-64 only inhibited the three lower molecular weight mucinases (C, D, and E in fig 3). Overall, these results are consistent with those of others and support the

Table 1 Effect of proteinase inhibitors on T vaginalis mucinases

\begin{tabular}{|c|c|c|c|c|c|c|}
\hline \multirow[b]{2}{*}{ Proteinase inhibitor } & \multirow[b]{2}{*}{ Inhibitor type } & \multicolumn{5}{|c|}{ Inhibition of mucinase } \\
\hline & & $A$ & $B$ & $C$ & $D$ & $E$ \\
\hline Aprotinin & serine & - & - & - & - & - \\
\hline Benzamide & serine & - & - & - & - & - \\
\hline PMSF & serine & - & - & - & - & - \\
\hline EDTA & metallo & - & - & - & - & - \\
\hline 1-10 phenanthroline & metallo & - & - & - & - & - \\
\hline E-64 & cysteine & - & - & + & + & + \\
\hline Iodoacetamide & cysteine & + & + & + & + & + \\
\hline Antipain & cysteine trypsin-like serine & + & + & + & + & + \\
\hline Leupeptin & cysteine trypsin-like serine & + & + & + & + & + \\
\hline TLCK & trypsin-like serine & + & + & + & + & + \\
\hline
\end{tabular}

$-=$ Proteinase activity not inhibited $+=$ proteinase activity inhibited. notion that trichomonad mucinases are cysteine-like.

We next wanted to determine the ability of mucinases to degrade mucin at relevant physiological $\mathrm{pH}$. It is known that vaginal $\mathrm{pH}$ in non-infected individuals is approximately 4.3, but can vary from below 4 to $\mathrm{pH} 7 .^{21} \mathrm{We}$, therefore, electrophoresed extracellular mucinases on BSM substrate gels and developed the gels in activation buffers ranging in $\mathrm{pH}$ from 4.5 to 7.0. Staining with Coomassie blue revealed that the above identified five mucinases were active over the whole $\mathrm{pH}$ range (fig 4).

Since trichomonads had different adherence levels to BSM and PSM, we tried to determine if mucin types had different susceptibility towards trichomonad mucinases. To address this question, trichomonad extracellular proteinases were electrophoresed on PSM and BSM substrate gels and developed as described before. Gels were then stained with Schiff's reagent, because PSM gels stained poorly with Coomassie. The pattern of proteinase activity visible on BSM substrate gels was identical when stained with Coomassie (fig 5, lanes 1 and 2) or Schiff's reagent (fig 5, lane 3). Surprisingly, the results in figure 5 show that trichomonad proteinases degrade BSM (fig 5, lane 3) but not PSM (fig 5, lane 4). To confirm that trichomonad proteinases are unable to degrade PSM, concentrated supernatants were incubated with BSM or PSM. At various time points, aliquots were removed and analysed by SDS-PAGE. Figure 6A shows that trichomonad proteinases degrade BSM to completion within 2 hours. Staining of gels with Coomassie stain gave identical results. In contrast, no degradation of PSM could be demonstrated in the same time period (fig 6B, lane). Lane 4 in figure $6 \mathrm{~B}$, however, demonstrates that degradation of PSM occurs, when the incubation period was extended to 24 hours. Staining of gels with silver or Coomassie stain, although resulting in very weak staining, appeared to confirm the results obtained with Schiff's stain.

TRICHOMONAD MUCINASES ARE ESSENTIAL FOR PENETRATION OF AN ARTIFICIAL MUCOUS LAYER To determine the involvement of trichomonad mucinases in breaching the mucous layer, we developed a mucous invasion assay. In this assay, trichomonads are placed in an invasion chamber, which has been coated with an agarose/mucin mixture. The chamber is then placed into a microtitre well, containing sufficient TYM serum medium to make contact with the invasion chamber. If parasites are able to penetrate the mucin layer, trichomonads can be expected to be found in the lower well. To determine the involvement of mucinases in the invasion process, this experiment was performed in the presence and absence of proteinase inhibitors. The data in table 2 show that live trichomonads easily traversed an artificial mucin layer composed of BSM or PSM, as live organisms were found in the lower chamber within 1 hour. In the presence of cysteine proteinase inhibitor, but 


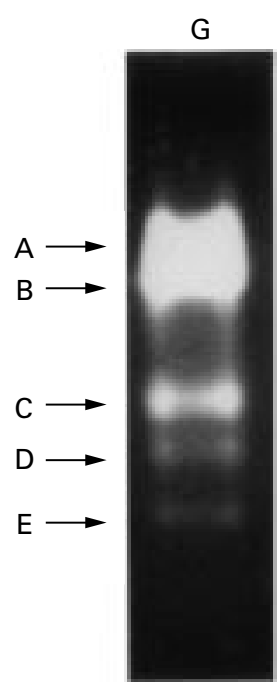

1
BSM

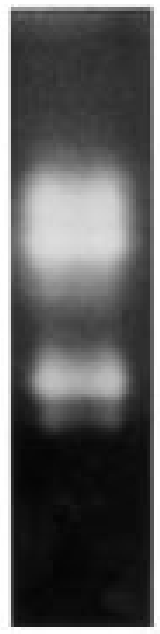

2

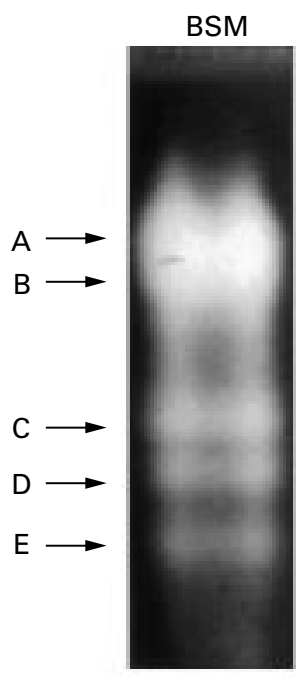

3

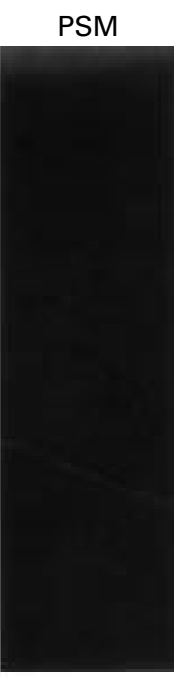

4

Figure 4 Proteolytic degradation of bovine submaxillary (BSM) and porcine stomach mucin (PSM) by trichomonad proteinases. Extracellular proteinases were separated by SDS-PAGE with copolymerised substrate. Gels were activated and developed as described in Materials and methods. Acrylamide gels were then stained with either Coomassie stain lanes 1 and 2) or Schiff's stain (lanes 3 and 4). As a reference the proteinase pattern with gelatin $(G)$ as the substrate is shown in lane 1.

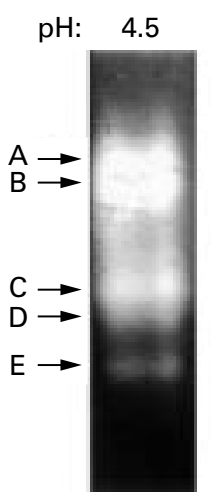

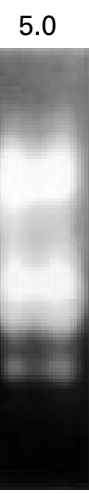

2

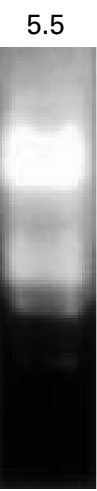

3

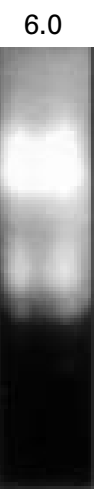

4
7.0

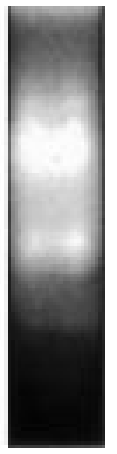

5

Figure 5 Activity of trichomonad mucinases at various $p H$ s as determined by substrate gel electrophoresis. Extracellular mucinases were separated by SDS-PAGE containing bovine submaxillary mucin as substrate. Gels were then activated in buffers at various $p H s$ in the presence of $1 \mathrm{mM}$ DTT. Gels were stained with Coomassie stain. Cleared areas represent digestion of the substrate.

not serine proteinase or metalloproteinase inhibitors, trichomonads were unable to traverse the mucin layer. This suggests that mucinase activity is necessary for crossing a mucin layer. It is noteworthy that a commercially available BSM contained a substance that inhibited trichomonad motility and that non-motile organisms were unable to breach the mucin barrier. For this reason BSM was purified by gel filtration before use in the invasion assay. This restored motility and the ability of trichomonads to breach the BSM mucin layer.

\section{Discussion}

The epithelial mucous layer is a formidable non-specific host defence. Mucin the principal glycoprotein of mucous secretion plays an important role in protection against microbial invasion, because of its heavy glycosylation and its ability to form a gel. ${ }^{23}$ The initial interaction between host and trichomonad occurs at the mucous surface, but it is unknown how $T$ vagi-
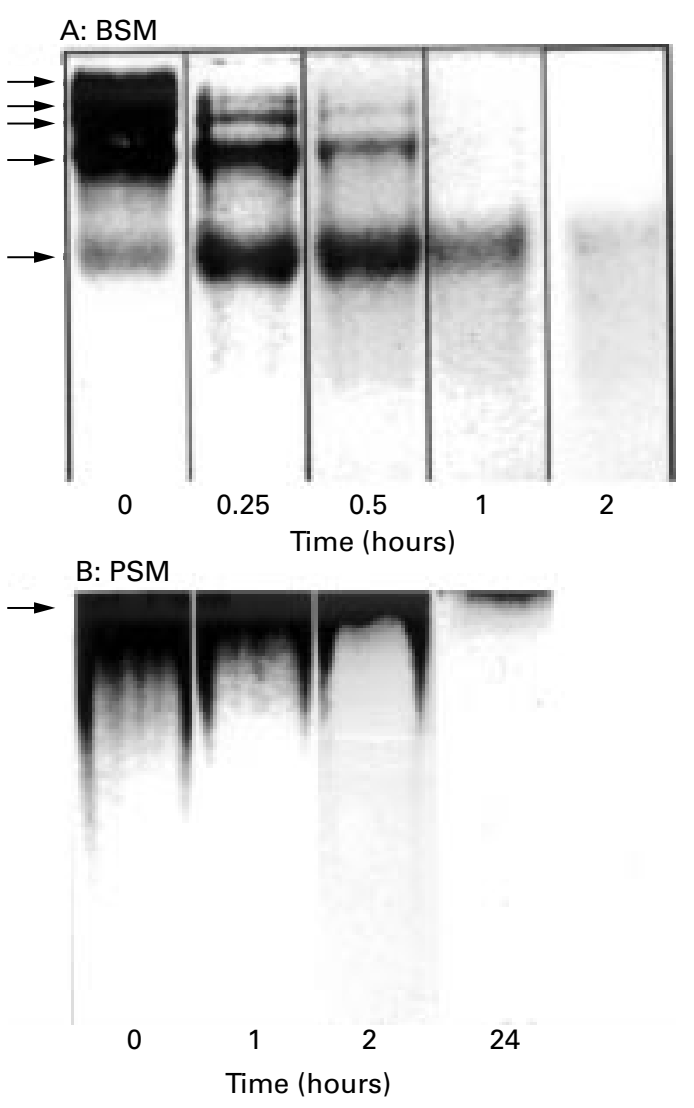

Figure 6 Time course degradation of bovine submaxillary mucin (BSM) (A) and porcine stomach mucin (PSM) (B). Extracellular trichomonad proteinases were incubated with BSM or PSM at $37^{\circ} \mathrm{C}$ for up to 24 hours in PBS containing $1 \mathrm{mM}$ DTT. At various times aliquots were removed and analysed by $S D S-P A G E$.

nalis circumvents this host barrier. Our results suggest that trichomonad penetration of the mucous layer requires adhesion, proteolytic cleavage of mucin and finally motility.

Our data demonstrate that $T$ vaginalis adheres to mucin (fig 1) and we suggest that adhesion to this host surface is prerequisite to colonisation of the host. Adherence to host surfaces is one of the prerequisites for infection of many pathogens and it may be especially important for mucosal pathogens. ${ }^{4}$ Microorganisms colonising mucosal surfaces always

Table 2 Effect of proteinase inhibitors on the ability of $T$ vaginalis to cross an artificial mucin layer

\begin{tabular}{lll}
\hline Chamber coating & Treatment & $\begin{array}{l}\text { Location of trichomonads } \\
\text { (upper chamber/lower well) }\end{array}$ \\
\hline Agarose & None & $+/-^{\star}$ \\
& PMSF & ND \\
& EDTA & ND \\
Agarose/BSM & TLCK & ND \\
& None & $+/+$ \\
& PMSF & $+/+$ \\
& EDTA & $+/+$ \\
Agarose/PSM† & TLCK & $+/-$ \\
& None & $+/+$ \\
& PMSF & $+/+$ \\
& EDTA & $+/+$ \\
Agarose/BSM $\ddagger$ & TLCK & $+/-$ \\
& None & $+/-$ \\
& PMSF & ND \\
& EDTA & ND \\
& TLCK & $+/-$
\end{tabular}

«Plus sign refers to trichomonads present and negative sign refers to trichomonads absent.

†Chromatographically purified mucin.

$\ddagger$ Commercial mucin, trichomonads are non-motile.

$\mathrm{ND}=$ not done. 
face the possibility of elimination from this site as a result of the flushing action of mucosal secretions. This point is well illustrated by studies that show that male ejaculate, including motile sperm, are removed from the vagina within a $12-24$ hour period. ${ }^{22}{ }^{23}$ In addition, it has been suggested that adherence of trichomonads to epithelial cells is also a strategy of this parasite to persist within the vagina. ${ }^{7}$ Clearly, adhesion to mucin then would provide micro-organisms, including trichomonads, with a mechanism to remain within the vaginal milieu for prolonged periods of time after coitus. Indeed, that adherence to mucin is an important aspect of infection is illustrated by the fact that a variety of pathogens, including Pseudomonas aeruginosa, ${ }^{24}$ Helicobacter pylori, ${ }^{25}$ Escherichia coli, ${ }^{26}$ Yersina enterocolitica, ${ }^{27}$ and the protozoan parasite Entamoeba histolytica ${ }^{28}$ bind mucin.

The ability of trichomonads to bind mucin, however, appears to serve two functions. Firstly, binding to mucin allows this protozoan to persist within the vaginal milieu. Secondly, adherence appears to be necessary for penetration of the mucous layer. This contention is supported by the observation of differential susceptibility of porcine mucin to proteinases in the presence or absence of metabolically active cells. In this study, we show that degradation of porcine mucin by soluble proteinases requires extended incubation periods. In contrast, porcine mucin degradation occurs rather quickly in the presence of live parasites as shown by the ability of trichomonads to traverse a porcine mucin layer within an hour of interaction. A possible explanation for these apparently contradictory results may be that binding to mucin allows for the build up of high local concentration of mucinase activity. Alternatively, binding may destabilise mucin molecules so that the parasite's mucinases can attack its peptide backbone.

Our data (fig 1) also suggest that binding to mucin is iron regulated. Threefold higher adherence levels to PSM were seen with low iron grown trichomonads when compared with trichomonads grown in high iron medium. Interestingly, trichomonad adherence to epithelial cells has also been shown to be iron regulated. ${ }^{7}$ In contrast with mucin adherence, epithelial cell adherence is upregulated under high iron conditions. In this case, coordinate transcriptional regulation of adhesin genes by iron contributed to elevated adherence levels. ${ }^{7}$ Whether mucin adherence mechanisms are also regulated by a transcriptional mechanism needs to be determined. Furthermore, the differential regulation of mucin adherence and cytoadherence, suggest that molecules involved in mucin adherence are distinct from cell adhesins. Interestingly, binding of mucin nevertheless interferes with cytoadherence in a dose dependent fashion (fig 2). Similarly, binding of mucin to $E$ histolytica also prevents amoebic attachment to host cells. ${ }^{28}$ The mode of cytoadherence inhibition, however, may be different in both organisms. E histolytica binds mucin through its adherence lectin and thus prevents binding to host cells. ${ }^{20}{ }^{28}{ }^{29}$ In contrast, trichomonad cell adhesins may be sterically blocked by mucin binding. Overall, these data and reports showing iron modulated expression of immunogens, ${ }^{15}$ lactoferrin receptors, ${ }^{15}$ cytoadherence, ${ }^{7}$ and complement resistance ${ }^{30}$ underscore the importance of environmental signals, especially iron, in the pathogenesis of trichomonosis.

The differential binding of trichomonads under low iron conditions to BSM and PSM was surprising and requires explanation. Most of the peptide backbone of mucin is inaccessible because of its heavy glycosylation. ${ }^{23}$ The glycosylation patterns of mucins vary from tissue to tissue and clearly from species to species. ${ }^{2}$ If trichomonads bind the sugar moieties of mucin, the difference in glycosylation pattern between PSM and BSM may account for the differential binding of trichomonads. Although no direct evidence for binding of the sugar moieties of mucin by trichomonads was obtained, it is interesting to note that Tritrichomonas mobilensis expresses a mucin binding lectin. ${ }^{31}$ An alternative explanation of the data is suggested by the finding that trichomonad proteinases rapidly degrade BSM but not PSM (fig 4, lanes 3 and 4). Trichomonads possibly adhere identically to PSM and BSM in our assay, but the rapid degradation of BSM allows the parasite to detach from the surface.

Although adherence to mucin would provide trichomonads with an initial foothold within the host, continued adherence to mucin would be detrimental as the parasite would be slowly washed out. Additionally, we show that mucin adherence interferes with cytoadherence (fig 2) an absolute requirement for parasite colonisation. Our demonstration of mucinolytic activity in trichomonal lysates and supernatants thus appears to allow the parasite to circumvent the negative consequences of mucin binding. Clearly, the demonstration of the proteolytic dependent crossing of an artificial mucous layer strongly suggests that cysteine proteinases allow trichomonads to detach and penetrate a mucous matrix. Furthermore, the observation that live trichomonads, in contrast with secreted proteinases, readily degrade PSM suggests that adherence and mucinolytic activity are intimately linked in the process of crossing a mucous layer. Indeed, the expression of mucinolytic activity and the ability to adhere to mucin appears to be a common theme among mucin crossing pathogens. ${ }^{24} 252732-34$

The proteinases responsible for the mucinolytic activity appear to be identical to previously described trichomonad cysteine proteinases. $^{6-121835}$ The evidence for identity is based on shared common characteristics of mucinases and previously described proteinases, such as: (i) activation by DTT, (ii) broad substrate specificity, (iii) activity over a broad range of $\mathrm{pH}$, (iv) identical inhibition profile, and (v) similar molecular weight. Some of these characteristics of mucinases make them uniquely adapted to the vaginal environment and may confer on the parasite the ability to invade and colonise the host under a variety of environmental conditions. For example, the vaginal $\mathrm{pH}$ can vary from 4 to 7.5 during the 
menstrual cycle. ${ }^{21}$ Mucinases with a broad $\mathrm{pH}$ range of activity may thus be advantageous to the parasite in such an unpredictable environment and allow for colonisation at any menstrual stage. Also, the broad substrate specificity of trichomonad mucinases may allow the parasite to inactivate a variety of defence molecules found in mucus. Besides mucin, the major antimicrobials found in mucus are lysozyme, immunoglobulins, and lactoferrin. ${ }^{36}{ }^{37}$ The proteolytic degradation of immunoglobulins ${ }^{11}$ and lactoferrin by trichomonad proteinases has been recently shown.

Trichomonad motility always has been thought to play an important role in pathogenesis, and indeed has been implicated to play a role in cytoadherence ${ }^{38}$ and chemotaxis. ${ }^{39}$ However, the significance of these findings for survival of trichomonads in vivo were uncertain. Serendipitously, we obtained evidence that motility is required for penetration of the mucous layer. Parasites added to invasion chambers coated with BSM were unable to cross into the lower chamber (table 2). Microscopic examination revealed that all trichomonads were immotile. Using chromatographically purified mucin in the invasion chamber restored motility and the ability of trichomonads to cross the mucin layer. Thus, it appears that motility is an important factor in the initial colonisation of a host.

Overall, this report implies a model of the initial interaction of trichomonads with the host. After deposition of the parasite on the mucosal surface, lectin-like adhesins anchor the trichomonad cell to the mucous layer. Adhesion is followed by the secretion of mucinases which solubilises the mucous matrix and detaches the parasite from the mucous layer. Flagellar movement, then allows the parasite to penetrate the solubilised matrix and colonise the underlying epithelial cells. It is interesting to note that although the details of mucous invasion by $T$ vaginalis and $E$ histolytica may differ, both organism only invade the underlying epithelial cells after comprising the integrity of the mucous layer. ${ }^{28} 29$

The interaction of trichomonads with mucin also suggests novel strategies to prevent transmission of this protozoan parasite. For example, a study showed that use of the spermicide nonoxyl-9 reduces transmission rates of $T$ vaginalis by $17 \% .^{40}$ Inclusion of inhibitors of trichomonad cysteine proteinases in nonoxynol-9 preparation may lower transmission rates even further. Clearly, the importance of cysteine proteinases in the initial steps of infection and their reported involvement in a variety of virulence properties such as adhesion, ${ }^{6}$ immune evasion, ${ }^{11}$ and nutrient acquisition ${ }^{35}$ provides support for the need to further investigate the structure, function, and role of the trichomonad cysteine proteinases in the pathophysiology of the disease.
This study was supported by a grant (G12-RR08124) from the Research Centers in Minority Institutions, National Center for Research Resources, NIH, and by a grant (S06-GM08012-25) from the Minority Biomedical Research Centers, NIH. We thank John F Alderete for his kind gift of $T$ vaginalis strains. We also thank Daniele Provenzano and John F Alderete for their suggestions and encouragement throughout this study. We are grateful to R Cabeza, S Das, and S Maier for their critical review of our manuscript.

1 Heine P, McGregor JA. Trichomonas vaginalis: a reemerging pathogen. Clin Obstet Gynecol 1993;36:137-44.

2 Rose MC. Mucins: structure, function, and role in pulmonary diseases. Am f Physiol 1992;263:L413-29.

3 Gerken TA. Biophysical approaches to salivary mucin structure, conformation and dynamics. Crit Rev Oral Biol Med 1993;4:261-70.

4 Abraham SN, Beachey EH. Host defenses against adhesion of bacteria to mucosal surfaces.In: Gallin JI, Fauci AS, eds. of bacteria to mucosal surfaces.In: Gallin JI, Fauci AS, eds. Advances in host defense mechanisms. Vol 4.
immunity. New York: Raven Press, 1985:63-88.

5 Alderete JF, Garza GE. Identification and properties of Trichomonas vaginalis proteins involved in cytoadherence. Infect Immun 1988;56:28-33.

6 Arroyo R, Alderete JF. Trichomonas vaginalis surface proteinase activity is necessary for parasite adherence to epithelial cells. Infect Immun 1989;57:2991-7.

7 Lehker MW, Arroyo R, Alderete JF. The regulation of the synthesis of adhesins and cytoadherence levels in the protozoan Trichomonas vaginalis. F Exp Med 1991;174: 311-18.

8 Coombs GH, North MJ. An analysis of the proteinases of Trichomonas vaginalis by polyacrylamide gel electrophoresis. Parasitology 1983;86:1-6.

9 Alderete JF, Newton E, Dennis C, et al. The vagina of women infected with Trichomonas vaginalis has numerous proteinases and antibody to trichomonad proteinases. Genitourin Med 1991;67:469-74.

10 Alderete JF, Provenzano D. The vagina has reducing environment sufficient for activation of Trichomonas vaginalis cysteine proteinases. Genitourin Med 1997:73:291-6.

11 Provenzano D, Alderete JF. Analysis of human immunoglobulin-degrading cysteine proteinases of Trichomonas vaginalis. Infect Immun 1995;35:903-9.

12 North MJ, Robertson CD, Coombs GH. The specificty of trichomonad cysteine proteinases analyzed using fluorogenic substrates and specific inhibitors. Mol Biochem Parasitol 1990;39:183-94.

13 Diamond LS. The establishment of various trichomonads of animal and man in axenic cultures. F Parasitol 1957;43:

14 Gorrell, TE. Effect of culture medium iron content on the biochemical composition and metabolism of Trichomonas vaginalis. $\mathcal{F}$ Bacteriol 1985;161:1228-30.

15 Lehker MW, Alderete JF. Iron regulates growth of Trichomonas vaginalis and the expression of immunogenic trichomonad proteins. Mol Microbiol 1992;6:123-32.

16 Laemmli UK. Cleavage of structural proteins during the assembly of the head of bacteriophage T4. Nature 1970;227:680-5.

17 Coley HM, Lewandowicz G, Sargent JM, et al. Chemosensitivity testing of fresh and continuous tumor cell cultures using lactate dehydrogenase. Anticancer Res 1997;17:231-

18 Neale KA, Alderete JF. Analysis of the proteinases of representative Trichomonas vaginalis isolates. Infect Immun 1990;58:157-62.

19 Heussen C, Dowdle EB. Electrophoretic analysis of plasminogen activators in polyacrylamide gels containing sodium dodecyl sulfate and copolymerized substrates. Anal Biochem 1980;102:196-202.

20 Belley A, Keller K, Grove J, et al. Interaction of LS174T human colon cancer cell mucins with Entamoeba histolytica: an in vitro model for colonic disease. Gastroenterology 1996;111:1484-92.

21 Stevens-Simon C, Jamison J, McGregor JA, et al. Racial variation in vaginal $\mathrm{pH}$ among healthy sexually active adolescents. Sex Transm Dis 1994;21:168-72.

22 Ricci LR, Hoffman SA. Prostatic acid phosphatase and sperm in the post-coital vagina. Ann Emerg Med 1982;11: $530-4$.

23 Wallace-Haagens MJ, Duffy BJ, Holtrop HR. Recovery of spermatozoa from human vaginal washings. Fertil Steril spermatozoa from

24 Reddy MS. Human tracheobronchial mucin: purification and binding to Pseudomonas aeruginosa. Infect Immun 1992;60:1530-5.

25 Tzouvelekis LS, Mentis AF, Makris AM, et al. In vitro binding of Helicobacter pylori to human gastric mucin. Infect Immun 1991;59:4252-4.

26 Sajjan SU, Forstner JF. Characteristics of binding of Escherichia coli serotype O157:H7 strain CL-47 to purified intestinal mucin. Infect Immun 1990;58:860-7.

27 Mantle M, Husar SD. Binding of Yersinia enterolytica to purified, native small intestinal mucins from rabbits and humans involves interactions with the mucin carbohydrate moeity. Infect Immun 1994;62:1219-27.

28 Chadee K, Petri WA, Innes DJ, et al. Rat and human colonic mucins bind to and inhibit adherence lectin of Entamoeba hucins bind to and inhibit adherence lectin

29 Chadee K, Ndarathi C, Keller K. Binding of proteolyticallydegraded human colonic mucin glycoproteins to the $\mathrm{Gal} / \mathrm{GalNAc}$ adherence lectin of Entamoeba histolytica. Gut 1990;31:890-5. 
30 Alderete JF, Provenzano D, Lehker MW. Iron mediates Trichomonas vaginalis resistance to complement lysis. Microbial Path 1995;19:93-103.

31 Babal P, Pindak DJ, Wells J, et al. Purification and characterization of a sialic acid-specific lectin from Tritrichomonas mobilensis. Biochem f 1994;299:341-6.

32 Colina AR, Aumont F, Deslauriers N, et al. Evidence for degradation of gastrointestinal mucin by Candida albican secretory aspartyl proteinase. Infect Immun 1996;64:4514-9.

33 Slomiany BL, Slomiany A. Mechanism of Helicobacter pylori pathogenesis: focus on mucus. $\mathcal{F}$ Clin Gastroenterol 1992;14:S114-21.

34 Mantle M, Rombough C. Growth in and breakdown of purified rabbit small intestinal mucin by Yersinia enterocopurified rabbit small intestinal mucin

35 North MJ. The characteristics of cysteine proteinases of parasitic protozoa. Biol Chem 1992;374:401-6.
36 Carlsted IH, Lindgren H, Sheehan JK. The macromolecularstructure of human cervical-mucus glycoproteins. Biochem F 1983;213:427-35.

37 Lamblin G, Roussel P. Airway mucins and their role in defense against micro-organisms. Respir Med 1993;87:421-

38 Krieger JN, Torian BE, Hom J, et al. Inhibition of Trichomonas vaginalis motility by monoclonal antibodies is associated with reduced adherence to HeLa cell monolayers. Infect Immun 1990;58:1634-9.

39 Styrt B, Sugarman B, Mummaw N, et al. Chemorepulsion of trichomonads by products of neutrophil oxidative metabotrichomonads by products of neutrop

40 Barbone F, Austin H, Louv WC, et al. A follow-up study of methods of contraception, sexual activity, and rates of trichomoniasis, candidiasis, and bacterial vaginosis. $A m \mathcal{F}$ Obstet Gynecol 1990;163:510-4.

\section{Joint Meeting of MSSVD and the ASTDA}

3-7 May 2000, Baltimore Marriott Inner Harbor Hotel, Baltimore, Maryland, USA

To mark the unique nature of the millennial year, for the first time in its 78 year history the Medical Society for the Study of Venereal Diseases will hold its Spring Meeting jointly with the American Sexually Transmitted Disease Association (President, Professor Julius Schacter). Our local host will be Professor Jonathan Zenilman of Johns Hopkins Medical School. The scientific programme will consist of plenary lectures and round table discussions, delivered by world authorities. There will also be oral and poster presentations of original work.

Further mailings will follow to MSSVD and ASTDA members. People who do not belong to either of these organisations and who would like to receive further information should contact: Dr Keith Radcliffe, Honorary Assistant Secretary, MSSVD (fax: +44 (0) 121-237 5729; email: k.w.radcliffe@bham.ac.uk). 